\title{
Atg6 is required for multiple vesicle trafficking pathways and hematopoiesis in Drosophila
}

\author{
Bhupendra V. Shravage ${ }^{1, *}$, Jahda H. Hill ${ }^{1,2, *, *}$, Christine M. Powers ${ }^{1}$, Louisa Wu ${ }^{2}$ and Eric H. Baehrecke ${ }^{1, \S}$
}

\begin{abstract}
SUMMARY
Atg6 (beclin 1 in mammals) is a core component of the Vps34 complex that is required for autophagy. Beclin 1 (Becn1) functions as a tumor suppressor, and Becn $1^{+/-}$tumors in mice possess elevated cell stress and p62 levels, altered NF- $\kappa B$ signaling and genome instability. The tumor suppressor function of Becn 1 has been attributed to its role in autophagy, and the potential functions of Atg6/Becn 1 in other vesicle trafficking pathways for tumor development have not been considered. Here, we generate Atg6 mutant Drosophila and demonstrate that Atg6 is essential for autophagy, endocytosis and protein secretion. By contrast, the core autophagy gene Atg1 is required for autophagy and protein secretion, but it is not required for endocytosis. Unlike null mutants of other core autophagy genes, all Atg6 mutant animals possess blood cell masses. Atg6 mutants have enlarged lymph glands (the hematopoietic organ in Drosophila), possess elevated blood cell numbers, and the formation of melanotic blood cell masses in these mutants is not suppressed by mutations in either p62 or NFKB genes. Thus, like mammals, altered Atg6 function in flies causes hematopoietic abnormalities and lethality, and our data indicate that this is due to defects in multiple membrane trafficking processes.
\end{abstract}

KEY WORDS: Autophagy, Endocytosis, Protein secretion, Drosophila

\section{INTRODUCTION}

Macroautophagy (autophagy) is an evolutionarily conserved catabolic process that is induced in response to cell stress, such as nutrient restriction, organelle damage and protein aggregation. During autophagy, double-membrane vesicles, known as autophagosomes, sequester cytoplasmic components, such as proteins and organelles, and deliver them to the lysosome for degradation (Mizushima and Komatsu, 2011). Protein turnover by the lysosome enables recycling of amino acids to be utilized for protein synthesis, and breakdown of damaged organelles prevents accumulation of toxic reactive oxygen species in the cell (Yang et al., 2006; Zhang et al., 2007). Autophagy has been implicated in many processes, including the mitigation of cell stress and genome instability, tissue remodeling during development, and clearance of intracellular pathogens (Berry and Baehrecke, 2007; Deretic, 2011; Karantza-Wadsworth et al., 2007).

Genetic screens in the yeast Saccharomyces cerevisiae identified autophagy-related (Atg) genes (Harding et al., 1995; Thumm et al., 1994; Tsukada and Ohsumi, 1993). These genes are required for autophagy and many are conserved in higher animals, including humans. A class III phosphoinositide 3-kinase (PI3K) complex, which includes the class III PI3K vacuolar protein sorting 34 (Vps34; also known as Pik3c3), the serine-threonine kinase Vps15 (p150 in mammals; also known as Pik3r4), and Atg6/Becn1 (also known as Vps30 in yeast), regulates autophagosome formation in yeast and mammals (Funderburk et al., 2010; Kihara et al., 2001b). The substrate of Vps34, phosphatidylinositol (PtdIns or PI), is

\footnotetext{
${ }^{1}$ Department of Cancer Biology, University of Massachusetts Medical School, Worcester, MA 01605, USA. ²Department of Cell Biology and Molecular Genetics, University of Maryland, College Park, MD 20742, USA.

*These authors contributed equally to this work

¥Present address: National Heart, Lung, and Blood Institute, National Institutes of Health, Bethesda, MD 20892, USA

§Author for correspondence (Eric.Baehrecke@umassmed.edu)
}

Accepted 11 January 2013 converted to PI 3-phosphate [PI3P], and this membrane-associated lipid is bound by proteins containing either FYVE or PX domains (Ellson et al., 2002; Stenmark et al., 2002). PI3P-containing membranes include autophagosome isolation membranes, which serve as precursors to double-membrane autophagosomes prior to membrane expansion (Kirisako et al., 1999; Simonsen et al., 2004). In addition to their functions in autophagy, Vps34 and PI3P also regulate sorting of hydrolases to the yeast vacuole and mammalian lysosome, endocytic trafficking, and potentially multiple other vesicle trafficking processes (Juhász et al., 2008; Thoresen et al., 2010).

Two distinct Atg6/Becn1 protein complexes have been described in yeast and mammals (Itakura et al., 2008; Kihara et al., 2001b; Liang et al., 2008). Atg6, Vps34 and Vps15 form a core complex, which recruits other proteins to modulate the specific biological function of this complex. The Atg6, Vps34 and Vps15 complex interacts with Atg14L to promote autophagosome formation, whereas it interacts with Uvrag/Vps38 to regulate vacuolar protein sorting. Recent work also indicates that the complex containing Uvrag is involved in ligand-receptor degradation and cytokinesis (Thoresen et al., 2010). S. cerevisiae and Caenorhabditis elegans Atg6 are also required for retrograde transport from endosomes to the Golgi complex (Ruck et al., 2011; Seaman et al., 1997). In addition, Rubicon, Ambra1 and Bif1 (Zbtb24) function as regulators of these complexes (Fimia et al., 2007; Matsunaga et al., 2009; Takahashi et al., 2007; Zhong et al., 2009).

The function of beclin 1 as a tumor suppressor has influenced our understanding of the role of autophagy in cancer. BECN1 is monoallelically deleted in sporadic breast, ovarian and prostate cancers (Aita et al., 1999), and allelic loss of Becn1 in mice leads to lymphomas and carcinomas (Qu et al., 2003; Yue et al., 2003). At the cellular level, Becn $1^{+/}$tumors have decreased autophagy, elevated cell stress and genome instability (Mathew et al., 2007). Moreover, decreased beclin 1 function in oncogene-expressing tissues is associated with the accumulation of the autophagy cargo binding protein p62 (SQSTM1), altered NFאB signaling, and inflammation (Mathew et al., 2009). Combined, these results 
indicate that the tumor suppressor function of beclin 1 is related to its role in autophagy, but do not consider the potential functions of Atg6/Becn1 in other vesicle trafficking pathways for tumor initiation and progression.

Drosophila has a single beclin 1 ortholog, Atg6, which shares $71 \%$ amino acid identity with the evolutionarily conserved domain of mammalian beclin 1, and 50\% overall identity. Atg6 protein interacts with Vps34 (Pi3K59F - FlyBase) in vivo, and coexpression of Atg6 with either Vps34 or Vps15 (ird1 - FlyBase) is sufficient to induce autophagy (Juhász et al., 2008). Both Vps34 and Vps 15 are required for starvation-induced autophagy and adult viability in Drosophila (Juhász et al., 2008; Lindmo et al., 2008; Wu et al., 2007). However, the lack of a null Atg6 mutant has precluded full functional analysis of the Vps34 complex in flies.

Here, we use gene targeting to generate a Drosophila Atg6 null mutant, and show that Atg6 mutant fat body cells exhibit defects in autophagy and endocytosis. In addition, we show that $\operatorname{Atg} 6, \operatorname{Vps} 34$ and Atgl function in protein secretion. Consistent with the role of beclin 1 as a tumor suppressor, loss of $A \operatorname{tg} 6$ caused over-production of blood cells, a failure in proper blood cell differentiation and the formation of melanotic blood cell masses.

\section{MATERIALS AND METHODS}

\section{Fly stocks and culture}

Flies were reared at $25^{\circ} \mathrm{C}$ on standard cornmeal/molasses/agar media. The following Drosophila melanogaster stocks were used: $P\{P Z\} A \operatorname{tg} 6^{00096}, y w$ hs-FLP ;FRT82B ubi-GFP, w;Sgs $43-G F P, y w$ hs-FLP; FRT42DGFPnls, $y w$ hs-FLP ;FRT42D mRFPnls, $y w$ hs-FLP; FRT80B mRFPnls, $y w$ hsFLP; FRT80B ubi-GFP, $y$ w eye-FLP1, w; Sgs $\triangle 3-G F P, y$ ws-FLP; actin $>c d 2>$ GAL4::UAS-dsRed, hmlGAL4::UAS-GFP, CgGAL4 (all from Bloomington Drosophila Stock Center); y w hs-FLP;CgGAL4 UASmCherryatg8a; FRT82B UAS-GFPnls, UAS-GFP-2x-FYVE, UASRab5-GFP, $y$ w hs-hflp; r4GAL4FRT82B UAS-mCherry, y $w$ hs-FLP; FRT80B Atg ${ }^{\triangle 3 D}$ (all from T. Neufeld, University of Minnesota, MN, USA; $y$ whs-FLP; FRT42DVps25n55 y+/CyO actin GFP (from A. Bergmann, University of Massachusetts Medical School, MA, USA); Atg6 ${ }^{I R}$ (TID 22123) (from Vienna Drosophila RNAi Center); Ref(2)P ${ }^{O D 2}, \operatorname{Ref}(2) P^{O D 3}$ (both from I. Nezis, University of Warwick, UK); w; rel $l^{e 20}, w ; D i f^{l} \mathrm{cn}$ $b w / C y O, w ; J 4 / C y O, d l^{I 5}$ (all from T. Ip); w;Atg ${ }^{d 77} / C y o$ actin-GFP, w; Atg7 d14/Cyo actin-GFP, $y^{l}$ Atg8a KG07569/FM7c-actin-GFP, $\operatorname{Atg} 13=\operatorname{Atg} 13^{D 74}$ (all from T. Neufeld); and FRT42D Vps32 ${ }^{G 5} /$ Cyo twistGal4 UAS-GFP (from D. Bilder, University of California, Berkeley, CA, USA).

\section{Larval staging}

For lymph gland analyses, larvae were obtained from collections of 4-hourold eggs and aged at $25^{\circ} \mathrm{C}$. Classification of third instar larvae was based on the number of teeth on the mandibular hooks (Bodenstein, 1965) and developmental age. For eye imaginal disc experiments, larvae were raised on food supplemented with $0.05 \%$ Bromophenol Blue (Maroni and Stamey, 1983). Stationary larvae with clear guts were used for dissection of eye imaginal discs from Atg6 and Vps mutants.

\section{Generation of Atg6 targeting construct}

The 'ends-out' gene disruption approach (Rong and Golic, 2000) was used to target the open reading frame (ORF) of Atg6 in the isogenic $w^{1118}$ parental line. The resultant strain contained a $w^{+}$mini-gene in place of the Atg6 ORF.

\section{RT-PCR}

RNA was collected from third instar larvae $(n=10)$ using Trizol Reagent (Invitrogen) and was treated with DNase. cDNA was generated from $1 \mu \mathrm{g}$ of RNA, using Superscript II Reverse Transcriptase (Invitrogen), following standard protocols. cDNA was used as PCR template, using the following primers to amplify Atg6 and flanking gene sequences: Atg6: 5'CGAGCAGCTGGAGAAGATTAG-3' and 5'-GCGTTGATCTCTGACCAGTC-3'; CG5991-RA: 5'-CATTGCCTAATTGTGTCCGC-3' and
5'-GGAGAATTGGCGCAAGTGAC-3'; CG5991-RB: 5'-GCACAGCGATACGGAAGCAA-3' and 5'-GGAGAATTGGCGCAAGTGAC-3'; CG5991-RC: 5'-GCCTCTTCGCATTTGACGAC-3' and 5'-GGAGAATTGGCGCAAGTGAC-3'; CG5986: 5' -GGCGATAACGCTTGCATCAC$3^{\prime}$ and $5^{\prime}$-CGTTGATATCCCGCAAACGG-3'. Quantitative real-time PCR was performed as described (Denton et al., 2009).

\section{Induction of mutant clones of cells}

Standard methods were used for the induction of mutant clones of cells (Xu and Rubin, 1993). To induce loss-of-function mutant cell clones, we used $y$ $w$ hsFlp; FRT42D Ubi-nlsGFP, $y$ w hsFlp; FRT42D mRFP-nls, $y$ w hsFLP; +; FRT80B Ubi-nlsGFP, y w hs-FLP; +; FRT80B Ubi-mRFP, y w hs-FLP; +; FRT82B Ubi-nlsGFP, y w hsFlp; CgGAL4; FRT82B UAS$m$ Cherry and $y w$ hsFlp; FRT42D Ubi-nlsGFP. Four-hour egg lays were maintained at $37^{\circ} \mathrm{C}$ for 1 hour to induce clones in the larval salivary glands, and 10 -hour egg lays were maintained at $37^{\circ} \mathrm{C}$ for 1 hour to induce clones in the larval fat body.

\section{Nutrient restriction}

Second and third instar larvae were fed $20 \%$ sucrose in PBS, pH 7.4, and maintained at $25^{\circ} \mathrm{C}$ for 4 hours.

\section{Texas Red-avidin assay}

To visualize endocytosis, the fat body was dissected from third instar larvae and incubated ex vivo with Texas Red-avidin (Invitrogen) diluted in Schneider's media to a concentration of $80 \mu \mathrm{g} / \mathrm{ml}$ for 20 minutes, then chased with $0.5 \%$ BSA in cold PBS for 10 minutes prior to overnight fixation in $4 \%$ formaldehyde. The tissue was washed three times (10 minutes per wash) with $0.1 \%$ Tween-20 in PBS and mounted in Vectashield (Vector Laboratories). Images were collected on a Zeiss AxioImager Z1 equipped with an Apotome. Images were acquired with Axiocam and processed using Zeiss Axiovision Suite 4.8 and Photoshop CS4 and Adobe Illustrator CS4 14.0.0.

\section{Immunostaining}

For fat body immunofluorescence experiments, tissues were dissected in PBS and fixed overnight in $4 \%$ formaldehyde in PBS, pH 7.4, at $4^{\circ} \mathrm{C}$. Following fixation, tissues were washed with $0.1 \%$ Triton X-100 in PBS (PBST) for 2 hours, then blocked in $0.5 \%$ BSA in PBST (PBSBT) for 2 hours at room temperature. Primary antibody incubations were performed overnight at $4^{\circ} \mathrm{C}$ in PBSBT followed by washing in PBSBT for 2 hours at room temperature. Secondary antibody was added at a dilution of 1:200, and tissues were incubated for 4 hours at room temperature. Following a series of short PBSBT washes, tissues were mounted in Vectashield with DAPI (Vector Laboratories). The following primary antibodies were used: rabbit anti-Ref (2)P (1:1000; I. Nezis) (Nezis et al., 2008), mouse antiP1/NimC1 (1:10; I. Ando) (Asha et al., 2003) and mouse anti-L1 (1:10; I. Ando) (Asha et al., 2003). Secondary antibodies from Invitrogen were used at 1:200: goat anti-rabbit Oregon Green 488, goat anti-rabbit Alexa Fluor 546, goat anti-mouse Alexa Fluor 546 and donkey anti-rat FITC.

For lymph gland analyses, larval fillets were prepared as previously described (Budnik et al., 2006), and lymph glands were dissected and stained as previously described (Lebestky et al., 2000).

For immunohistochemistry of paraffin sections, third instar larvae were fixed and dehydrated for histology according to published methods (Muro et al., 2006). Histological sections were de-waxed with a series of xylene washes, and rehydrated through a series of decreasing percentage ethanol washes. Following rehydration, antigen retrieval was performed by heating slides in $10 \mathrm{mM}$ sodium citrate, $\mathrm{pH}$ 6.0. Specimens were blocked in 5\% non-fat dry milk containing 1\% BSA and horse serum (Vector Laboratories). Rabbit anti-GFP (Novus) antibody was used at 1:500. The Vectastain Elite $\mathrm{ABC}$ Kit (Vector Laboratories) was used for immunohistochemical detection, and the signal was visualized by diaminobenzadine staining. Tissue was counterstained with Weigert's Hematoxylin and Permount mounting media was applied. Images were collected on a Zeiss Axiophot microscope. Images were minimally processed using Adobe Photoshop CS4. 


\section{Hemocyte quantification}

Individual third instar larvae of similar age ( \pm 3 hours) were bled into $20 \mu \mathrm{l}$ of PBS. From this, $10 \mu \mathrm{l}$ was loaded onto a standard hemocytometer and the average number of cells per milliliter was calculated for 20 animals per genotype. A one-tailed Student's $t$-test was used to determine statistical significance.

\section{Quantification and statistical analyses}

Zeiss Image Measurement Software was used for the quantification of Atg8a, Ref(2)P and Rab5 puncta, as well as the eye-antennal disc area and the lymph gland area. Statistical analyses were performed using GraphPad prism software. Student's $t$-test for two samples assuming unequal variances was used to determine the statistical significance of the data.

\section{Transmission electron microscopy}

Tissues were dissected and fixed overnight at $4^{\circ} \mathrm{C}$ in $4.0 \%$ paraformaldehyde, $2.0 \%$ glutaraldehyde, $1 \%$ sucrose and $0.028 \% \mathrm{CaCl}_{2}$ in $0.1 \mathrm{M}$ sodium cacodylate, $\mathrm{pH} 7.4$, thoroughly washed in cacodylate buffer, post-fixed in $2.0 \%$ osmium tetroxide for 1 hour, and embedded in SPIpon/Araldite resin (Polysciences) according to manufacturer's recommendations. Ultrathin sections $(80 \mathrm{~nm})$ were stained with uranyl acetate and lead citrate before examination in a Philips CM10 transmission electron microscope.

\section{Protein secretion assay}

Salivary glands were dissected from control and mutant animals at 4 hours after puparium, fixed for 30 minutes in $4 \%$ paraformaldehye in PBS, washed three times (5 minutes per wash) in PBS, and mounted in Vectashield with DAPI.

\section{RESULTS}

\section{Atg6 is required for autophagy in Drosophila}

A transposable P-element, $P\{P Z\}$ Atg $6^{00096}$, is located in the 5' untranslated regions of Atg6 and the neighboring gene CG5991 (Fig. 1A). Starvation-induced autophagy was not consistently altered in the fat body of homozygous $P\{P Z\} A \operatorname{tg} 6^{00096}$ larvae (Scott et al., 2004). Based on this result and the fact that $P\{P Z\} A \operatorname{tg} 6^{00096}$ does not disrupt the Atg6 ORF, we generated an Atg6 mutant using a gene-targeting approach (Rong and Golic, 2000).

Homologous recombination was used to replace the Atg6 ORF with a $w+$ mini-gene (Fig. 1A). RT-PCR confirmed the absence of Atg6 RNA in homozygous mutant third instar larvae, whereas the RNA levels of neighboring genes were not altered in the homozygous mutant $\operatorname{Atg} 6$ (hereafter termed as $\operatorname{Atg} 6^{l}$ ) mutant larvae (Fig. 1B). In addition, PCR was used to confirm the presence of a $w+$ mini-gene in the Atg6 genomic locus (data not shown). Both homozygous $A \operatorname{tg} 6^{1}$ and animals transheterozygous for $A \operatorname{tg} 6^{1}$ and the $D f(3 R)$ Exel 6197 deficiency for this region died during the late third larval instar and early pupal stages of development (supplementary material Fig. S1A; data not shown). Significantly, expression of a UAS-GFP-Atg6 transgene under the control of a ubiquitously expressed actin-GAL4 driver rescued the lethality of $\operatorname{Atg} 6^{1}$ homozygous mutants (supplementary material Fig. S1A). In addition, $A \operatorname{tg} 6^{1}$ complements $P\{P Z\} A \operatorname{tg} 6^{00096}$ even though this is a lethal P-element insertion, indicating that $P\{P Z\} A \operatorname{tg} 6^{00096}$ is not an Atg6 mutant. These data indicate that we have isolated a loss-offunction Atg6 mutant.

Atg6, Vps 15 and Vps34 are core components of all known Vps34 complexes (Funderburk et al., 2010), and loss of either Vps34 or Vps15 inhibits starvation-induced autophagy in the larval fat body of Drosophila (Juhász et al., 2008). To determine whether Atg6 is required for starvation-induced autophagy, we monitored the localization of the autophagosome marker mCherry-Atg8a in larval fat body. Atg8a, the Drosophila ortholog of mammalian LC3 (microtubule-associated protein 1 light chain 3), displays diffuse
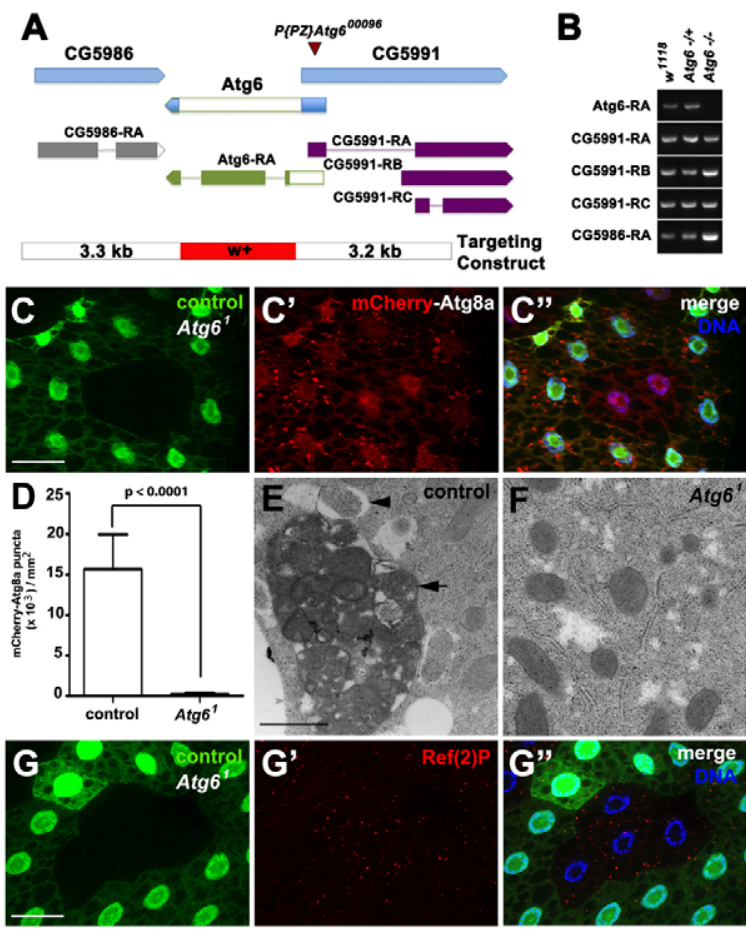

Fig. 1. Atg6 is required for autophagy. (A) Atg6 genomic locus and donor targeting construct (not to scale). The donor construct consisted of a $w+$ mini-gene flanked by $3.5 \mathrm{~kb}$ of genomic sequence (blue) from each side of Atg6. Flp recombination target (FRT) sites and I-Scel endonuclease recognition sites were present on each side of flanking sequence, to facilitate double-stranded break and homologous recombination at the target site. The resultant flies contained the $w+$ minigene (red) in place of the Atg6 ORF (white). (B) RT-PCR of Atg6 and flanking gene transcripts indicates knockdown of Atg6 transcript expression in homozygous Atg6 third instar larvae, and maintenance of flanking gene RNA levels.

(C-C') mCherry-Atg8a puncta reflect starvation-induced autophagosome formation in control (GFP-positive) larval fat body cells, whereas

cytoplasmic mCherry-Atg8a localization reflects a defect in autophagy in Atg6 ${ }^{1}$ (GFP-negative) fat body cells ( $n=10$ ). (D) Quantification of mCherryAtg8a puncta in control and Atg $6^{1}$ mutant fat body cells following starvation. A two-tailed $t$-test was used for statistical analysis and the $P$ value relative to control was $8.98 \times 10^{-8}$. Error bars represent s.d. (E,F) TEM images reveal abundant autophagosomes (arrowhead) and autolysomes (arrow) in control (E) but not in (F) Atg6 $6^{1}$ mutant fat body cells following 4 hours of starvation. (G-G') Ref(2)P accumulated in Atg6 $6^{\prime}$ (GFP negative) fat body cells, indicative of defective autophagy, compared with control

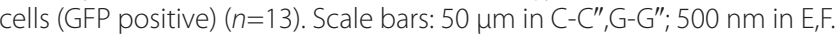

cytoplasmic localization in the fat body of feeding Drosophila larvae, but becomes incorporated into autophagosome membranes during starvation and is visualized as punctate spots in the cytoplasm (Scott et al., 2004). We utilized FLP recombinasemediated recombination at FLP recombination target (FRT) sites to generate $\operatorname{Atg} 6^{1}$ mitotic mutant cell clones in the fat body, resulting in tissue composed of control (either wild type or heterozygous $\operatorname{Atg} 6^{1} /$ wild type) and homozygous $\operatorname{Atg} 6^{1} / \operatorname{Atg} 6^{1}$ mutant cells. Following 4 hours of starvation, control fat body cells, which were marked by the presence of green fluorescent protein (GFP) contained several mCherry-Atg8a puncta, whereas homozygous $\operatorname{Atg} 6^{1}$ mutant cells (lacking GFP) displayed diffuse localization of mCherry-Atg8a (Fig. 1C-C",D).

Two additional approaches were used to determine the influence of Atg6 function on autophagy. We used transmission electron 
microscopy (TEM) to investigate $\operatorname{Atg} 6^{1}$ mutants at the ultrastructural level. Following 4 hours of starvation, many autophagosomes (Fig. 1E, arrowhead) and autophagolysosomes (Fig. 1E, arrow) were observed in control fat body cells, whereas homozygous $\operatorname{Atg} 6^{1}$ mutant fat body cells lacked these autophagic structures (Fig. 1F). Ref(2)P is the Drosophila ortholog of p62 (SQSTM1) and is known to bind ubiquitylated substrates and aid in their recruitment into autophagosomes to be targeted for degradation (Nezis et al., 2008). Homozygous $\operatorname{Atg} 6^{1}$ mutant cells lacking GFP accumulated $\operatorname{Ref}(2) \mathrm{P}$ compared with neighboring control fat body cells (Fig. 1G-G"; supplementary material Fig. S1B). Importantly, the accumulation of $\operatorname{Ref}(2) \mathrm{P}$ aggregates could be rescued by expressing an Atg6 transgene in the $\operatorname{Atg} 6^{1}$ mutant cells (supplementary material Fig. S1C-C'). These results indicate that Atg6 is required for autophagy in vivo.

\section{Atg6 functions in multiple vesicle trafficking processes}

Vps34 is required for the formation of PI3P in Drosophila and other species (Juhász et al., 2008; Lindmo and Stenmark, 2006). To examine the influence of Atg6 function on PI3P, we used a transgenic reporter consisting of GFP fused to the FYVE domain of hepatocyte growth factor regulated tyrosine kinase substrate (Hrs)
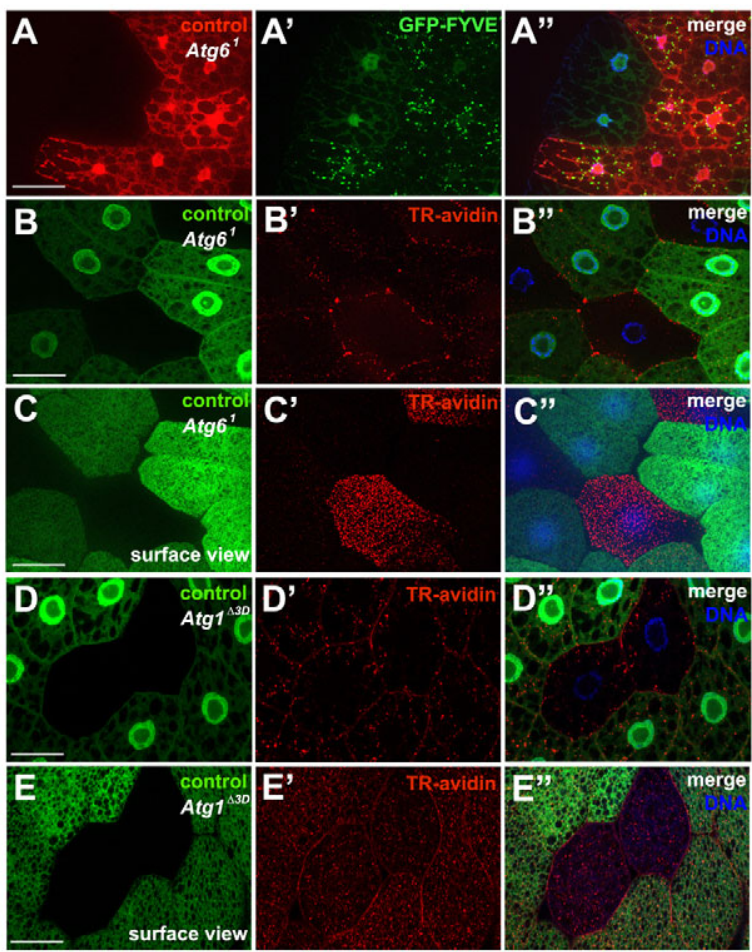

Fig. 2. Loss of Atg6 leads to defects in PI3P formation and endocytosis. $\left(\mathbf{A}^{\left.-\mathbf{A}^{\prime \prime}\right)}\right.$ GFP-FYVE localizes to perinuclear puncta, reflecting Vps34 activity and PI(3)P formation in control (mCherry-positive) fat body cells, but remains cytoplasmic in Atg $6^{1}$ mutant (mCherry-negative) fat body cells $(n=24)$. ( $\left(\mathbf{B}-\mathbf{B}^{\prime \prime}\right)$ Texas Red-avidin is endocytosed by control (GFPpositive) fat body cells, but is largely excluded from Atg $6^{1}$ mutant cells (GFP negative). (C-C') Surface view of the fat body depicted in B showing accumulation of Texas Red-avidin at the surface of the Atg6 ${ }^{1}$ cells but not in control cells ( $n=13)$. ( $\left(\mathbf{D}-\mathbf{D}^{\prime \prime}\right)$ Texas Red-avidin is endocytosed in control (GFP-positive) fat body cells, as well as in Atg ${ }^{\triangle 3 \mathrm{D}}$ mutant cells (GFP negative). (E-E') Surface view of the fat body depicted in D showing Texas Red-avidin in both control and Atg $1^{\triangle 3 D}$ mutant cells $(n=10)$. Scale bars: $50 \mu \mathrm{m}$.
(Gillooly et al., 2000). GFP-FYVE localized to punctate structures in the cytoplasm of control (red) cells of the larval fat body (Fig. 2A$\mathrm{A}^{\prime \prime}$ ). By contrast, homozygous $\operatorname{Atg} 6^{1} / \operatorname{Atg} 6^{1}$ mutant cells (lacking red) had no detectable GFP-FYVE puncta (Fig. 2A-A").

Vps34 is required for endocytosis in Drosophila (Juhász et al., 2008). To test whether Atg6 functions in endocytosis, uptake of Texas Red (TR)-avidin was used to monitor fluid-phase endocytosis in larval fat body. Control (GFP-positive) cells contained TRavidin-positive puncta throughout the cytosol, whereas homozygous Atg $6^{1}$ mutant (GFP-negative) cells possessed little to no endocytic tracer (Fig. 2B-B") and instead TR-avidin was often more abundant on the surface of these mutant cells (Fig. 2C-C").

Rab5, a small GTPase of the Ras superfamily, is associated with endosomes and functions as a key regulator of vesicle trafficking (Wucherpfennig et al., 2003). To monitor Rab5 localization in fat body cells, we used a GFP-Rab5 transgenic reporter. In control fat body cells, GFP-Rab5 localizes to the plasma membrane and has characteristic puncta with perinuclear localization. In cells with RNAi knockdown of $\operatorname{Atg} 6\left(\operatorname{Atg} 6^{I R}\right)$, GFP-Rab5 localized to the plasma membrane in most fat body cells; however, the perinuclear localization was significantly reduced (supplementary material Fig. S2A-D). These data suggest that in larval fat body cells, Atg6 is required for either recruitment of or stable association of Rab5 with the perinuclear endosomal compartment.

To determine whether the endocytosis phenotypes observed in Atg6 and Vps34 mutant cells are due to defects in autophagy, we performed the TR-avidin uptake assay in Atg1 mutant fat cells. Atg1 is a kinase and a core component of the autophagy pathway that is both necessary and sufficient for inducing autophagy (Scott et al., 2007). Both control (GFP-positive) cells and homozygous $\operatorname{Atg} 1^{\triangle 3 D}$ (GFP-negative) mutant cells contained TR-avidin-positive puncta throughout the cytosol (Fig. 2D-D"). Furthermore, TR-avidin did not accumulate at the surface of these cells (Fig. 2E-E"), indicating that Atg1 function is not required for fluid-phase endocytosis. These results indicate that Atg6, but not Atg1, is required for fluid-phase endocytosis in vivo.

Endosomal sorting complex required for transport (ESCRT) proteins are required for recruitment of ubiquitylated cargo proteins to the endosome, sorting to multivesicular bodies (MVBs), and subsequent degradation by the lysosome (Henne et al., 2011). Like Atg6 mutant cells, mutations in either Vps25 (ESCRT-II) or Vps32 (ESCRT-III; $s h r b$ - FlyBase) (GFP-negative cells) suppress TRavidin fluid-phase endocytic tracer uptake compared with control cells (GFP-positive) (supplementary material Fig. S3A-D"). Loss of ESCRT components in the developing Drosophila eye impairs MVB formation and leads to defects in receptor degradation that cause tissue overgrowth (Fig. 3A,D) (Herz et al., 2006; Herz et al., 2009; Thompson et al., 2005; Vaccari and Bilder, 2005; Vaccari et al., 2009). By contrast, eye imaginal disc tissue isolated from homozygous $\operatorname{Atg} 6^{1}$ animals at the same third larval instar stage is normal in size (Fig. 3B). Although Atg6 and ESCRT component double mutant eye tissue is disorganized, loss of Atg6 significantly suppressed the overgrowth of either $\operatorname{Vps} 25$ or $V p s 32$ mutant eye tissue (Fig. 3C,E,F). Combined, our data indicate that Atg6 is required for fluid-phase endocytosis, and suggest the possibility that an important relationship might exist between Atg6 and the ESCRT pathway.

Recent work has implicated autophagy in protein secretion (Deretic et al., 2012), but direct genetic analyses of the role of key autophagy regulatory factors, including Atg6, in protein secretion within animals are lacking. The Drosophila larval salivary gland secretes large quantities of glue proteins in response to steroid at 

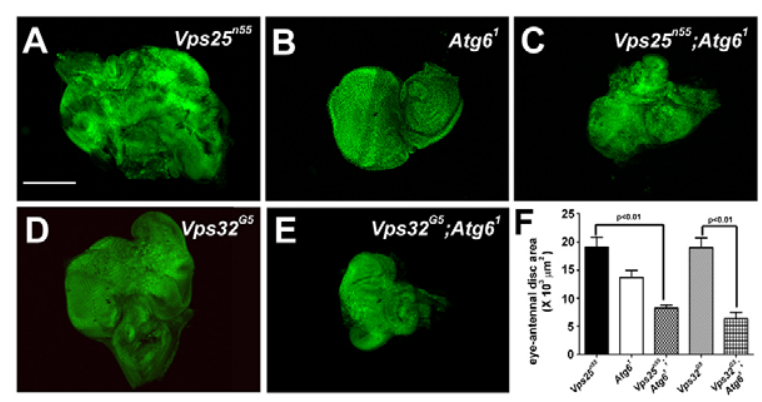

Fig. 3. Atg6 suppresses the Vps25 and Vps32 mutant eye phenotypes. (A) Vps $25^{n 55}$ mutant clone cells (GFP-negative) in late third instar eye imaginal discs cause non-autonomous over-proliferation of neighboring (GFP-positive) cells leading to overgrowth of the developing eye tissue ( $n=11$ ). (B) Homozygous Atg $6^{1}$ mutant late third larval instar eye imaginal discs appear normal in size and morphology (DAPI is shown in green, $n=10$ ). (C) Homozygous loss of Atg6 suppressed overgrowth of the eye caused by Vps 25 mutant cells (GFP negative) $(n=6)$. (D) Vps32 $2^{G 5}$ mutant clones (GFP-negative) in late third instar eye imaginal disc cause non-autonomous overproliferation of neighboring (GFP-positive) cells leading to overgrowth of the developing eye tissue $(n=11)$. (E) Vps32 $2^{\mathrm{G} 5}$ mutant clones (GFP negative) in $A \operatorname{tg} 6^{1}$ mutant eye disc leads to suppression of overgrowth of the eye discs $(n=10)$. (F) Quantification of eye-antennal disc area in each of the genotypes depicted in A-E. A twotailed $t$-test was used for statistical analyses. The $P$-value between Vps $25^{n 55}$ and Vps25 $5^{n 55}$; Atg $6^{1}$ was $6.6 \times 10^{-6}$ and the P-value between Vps32 $2^{G 5}$ and $V p s 32^{G 5}$; Atg $6^{1}$ was $1.97 \times 10^{-5}$. Error bars represent s.d. Scale bar: $50 \mu \mathrm{m}$.

the end of larval development. A transgenic fusion of the secreted glue protein Sgs3 and GFP proteins provides an excellent means to follow protein secretion in this tissue in vivo (Biyasheva et al., 2001). Both control (Fig. 4A, $\mathrm{A}^{\prime}$ ) and homozygous Atg $6^{1}$ mutant animal (Fig. 4C, $\mathrm{C}^{\prime}$ ) salivary glands are able to synthesize glue protein in the salivary glands based on the presence of GFP. By 4 hours after puparium formation, control animals had secreted most of the glue protein, based on the absence of GFP (Fig. 4B, B'), whereas homozygous $A \operatorname{tg} 6^{1}$ mutant animals retained GFP, suggesting that they have a protein secretion defect (Fig. 4D, D'). It is possible that this $\operatorname{Atg} 6^{l}$ mutant animal defect is caused by a failure to arrest protein synthesis that is associated a general delay in development. To address this possibility, homozygous $\operatorname{Atg} 6^{1}$ mutant clones of cells (mCherry negative) were produced in salivary glands. These Atg6 mutant cells retained Sgs3-GFP, whereas neighboring control cells (mCherry positive) were devoid of GFP reporter (Fig. 4E-E"). Furthermore, Sgs3-GFP was also retained in salivary gland cells homozygous for $V p s 34^{d m 22}$ (a null allele of Vps34, mRFP negative) as well as in cells with RNAi knockdown of $V p s 34$, whereas neighboring control cells (mRFP positive) lacked Sgs3-GFP (Fig. 3F-F"; data not shown). Interestingly, $\operatorname{Atg} 1^{\triangle 3 D}$ mutant cells also retained Sgs3-GFP, whereas neighboring control cells (mRFP positive) secreted Sgs3-GFP (Fig. 3G-G"). These data suggest that Atg6, Vps34 and Atg1 are each required for salivary gland protein secretion.

\section{Loss of Atg6 leads to melanotic blood cell mass formation}

Whereas the parental control $w^{1118}$ and heterozygous $\operatorname{Atg} 6^{1} /$ wildtype animals exhibited no obvious phenotypes, all homozygous Atg6 $6^{l}$ mutant larvae displayed a striking melanotic blood cell mass phenotype (visible as black masses) (Fig. 5A-C). Although single melanotic blood cell masses are often present in $\operatorname{Atg} 6^{I}$ mutant larvae

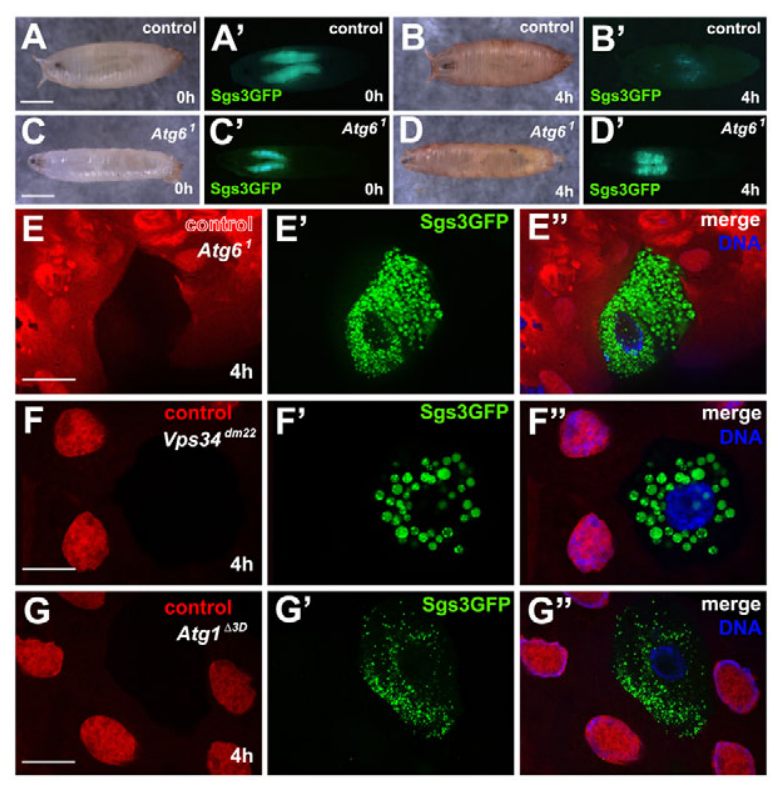

Fig. 4. Loss of Atg6 leads to defects in protein secretion.

$\left(\mathbf{A}, \mathbf{A}^{\prime}\right)$ Control pupa (0 hour) showing expression of Sgs $\triangle 3$-GFP in salivary glands $(n=10)$. (B, $\left.\mathbf{B}^{\prime}\right)$ Four hours later the same pupa as in A lacked Sgs $\triangle 3-G F P .\left(C_{-}-D^{\prime}\right)$ Homozygous Atg $6^{1}$ mutants possess Sgs $\triangle 3-G F P$ protein at 0 hour $\left(C-C^{\prime}\right)(n=12)$, but are unable to secrete it 4 hours after puparium formation (D-D'). (E-E') Control cells (mCherry positive) are able to secrete Sgs3 $\triangle 3$-GFP whereas homozygous Atg $6^{1}$ mutant cells (mCherry negative) retain Sgs $\triangle 3-G F P$ in the cytoplasm $(n=12)$. (F-F") Control cells (mCherry positive) secrete Sgs $\triangle 3$-GFP whereas Vps34 ${ }^{d m 22}$ mutant cells retain Sgs $\triangle 3$-GFP granules in the cytoplasm ( $n=11)$. (G-G') Control cells (mCherry positive) secrete Sgs $\triangle 3-G F P$ whereas $A \operatorname{tg} 1^{\triangle 3 D}$ expressing cells retain Sgs $\triangle 3$-GFP granules in the cytoplasm ( $n=10)$. Scale bars: $1 \mathrm{~mm}$ in A,C; $50 \mu \mathrm{m}$ in $\mathrm{E}-\mathrm{G}$.

(Fig. 5C), many homozygous mutant animals possess multiple melanotic blood cell masses (Fig. 6B). Significantly, this homozygous Atg6 mutant melanotic blood cell tumor phenotype was completely rescued by ubiquitous expression of Atg6 (Fig. 5D).

We investigated whether mutations in other core autophagy genes results in melanotic blood cell mass formation. All homozygous Atg7 and Atg13 mutant animals lack melanotic blood cell masses (supplementary material Fig. S4A; data not shown). Although 18\% of Atg8a ${ }^{K G 07569}$ mutant pupae possess melanotic masses (supplementary material Fig. S4A-C), such blood cell masses were not observed in Atg8 mutant larvae. Combined, these data indicate that Atg6 mutants are different from other autophagy mutants in their predisposition to the formation of melanotic blood cell masses.

We investigated whether blood cells are the source of melanotic masses in Atg6 mutant larvae. Blood cell-specific hemolectin $\left(\mathrm{hml}\right.$ ) $-\mathrm{GAL} 4$ was used to drive GFP expression in parental $w^{1118}$ control, heterozygous $\operatorname{Atg} 6^{1} /$ wild-type and homozygous $\operatorname{Atg} 6^{1}$ mutant larvae. Immunohistochemical analyses of paraffin sections with a GFP antibody revealed that these masses were indeed composed of blood cells in homozygous $\operatorname{Atg} 6^{1}$ mutants, whereas significantly fewer blood cells were observed in either $w^{1118}$ or $\operatorname{Atg} 6^{1} /$ wild-type control animals (Fig. 5E-G). Quantification of blood cells revealed that $\operatorname{Atg} 6^{1} /$ wild-type animals contained approximately twice as many blood cells as parental $w^{1118}$ larvae, and homozygous $\operatorname{Atg} 6^{1} / \operatorname{Atg} 6^{1}$ mutants contained nearly ten times as many blood cells as control $w^{1118}$ animals (Fig. 5H). These data indicate that loss of Atg6 results in an increase in blood cell 

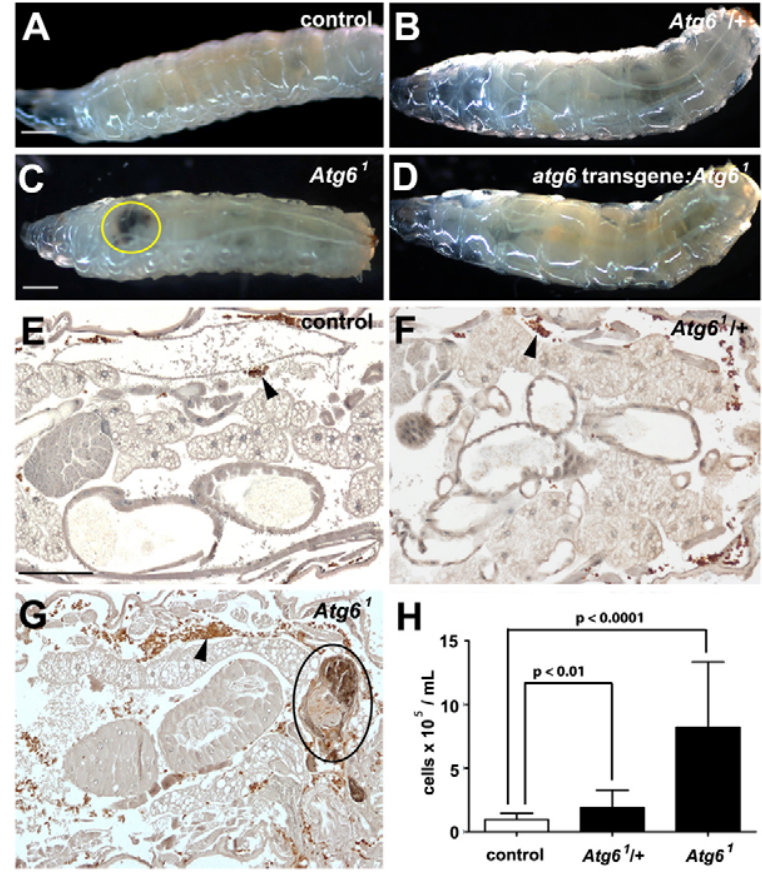

Fig. 5. Loss of Atg6 leads to melanotic blood cell mass formation.

(A-D) White-light images of wandering third instar larvae. Parental control $w^{1118}$ (A) and heterozygous Atg6 ${ }^{1}$ (B) larvae are phenotypically similar. Homozygous Atg6 $6^{1}$ mutant larvae (C) contain melanotic blood cell masses (yellow ring), and this phenotype is rescued by ubiquitous expression of a GFP-Atg6 transgene (D). (E-G) Hemocytes were visualized by immunohistochemistry in third instar larvae expressing GFP, driven by hm/ $\triangle$-GAL4 (arrowheads). Histological sections of $w^{1118}$ (E) and Atg $6^{1}$ heterozygous (F) larvae contain few hemocytes (brown) and no visible melanotic masses, whereas sections of homozygous Atg6 mutant larvae $(\mathrm{G})$ reveal many hemocytes, which surround melanotic masses (black ring). (H) Quantification of hemocytes from $w^{1118}$, Atg $^{1} /+$ and Atg $6^{1} /$ Atg $^{1}$ larvae revealed a significant increase in hemocyte number in both heterozygous and homozygous Atg6 mutant larvae compared with control $w^{1118}$ larvae. For each genotype, $n=20$ animals. Error bars represent s.e.m. A one-tailed $t$-test was used for statistical analysis and $P$ values relative to $W^{1118}$ are: $A \operatorname{tg} 6^{1} /+, P=0.004 ; A \operatorname{Atg} 6^{1} / \operatorname{Atg}^{1}, P=2.6 \times 10^{-6}$. Scale bars: $500 \mu \mathrm{m}$ in $\mathrm{A}, \mathrm{C} ; 100 \mu \mathrm{m}$ in $\mathrm{E}$.

numbers, and like beclin 1 mutant mice, loss of Atg6 causes an increase in the number of circulating blood cells.

Two approaches were taken to determine whether the melanotic mass phenotype is blood cell autonomous. First, we expressed UAS-Atg6 using two different blood cell-specific drivers, either hml-Gal4 or croquemort-Gal4, in $\operatorname{Atg} 6^{1}$ mutant animals. Expression of the Atg6 transgene using these approaches failed to rescue the phenotype, suggesting that ectopic blood cell masses are either due to a blood cell-independent effect of Atg6 or that these Gal4 drivers are expressed too late during blood cell development to rescue the mutant phenotype (data not shown). Second, we induced Atg6 loss-of-function mutant cell clones in lymph glands using $h s$-FLP. Although these animals possess melanotic masses (data not shown), they also have Atg6 mutant cell clones in other tissues. Thus, we cannot conclude that the Atg6 melanotic mass phenotype is blood cell lineage specific.

The current model for beclin 1 function during tumor progression suggests that decreased autophagy leads to elevated p62 signaling and activation of the NFKB pathway (Mathew et al., 2009). In addition, the NFkB pathway has been implicated in the formation of
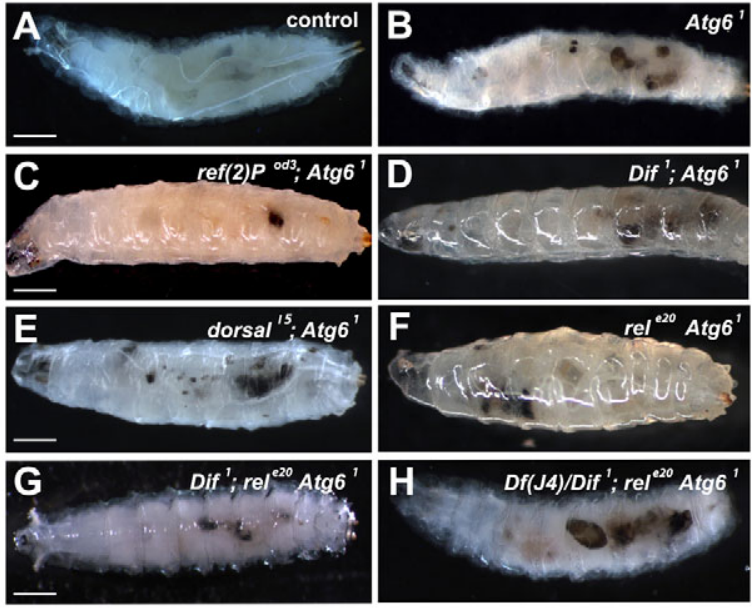

Fig. 6. Ref(2)P and NFKB are not required for melanotic mass formation in Atg $^{1}$ mutant animals. (A) Control late third instar larvae lack melanotic masses. (B) All homozygous Atg $6^{1}$ mutant late third instar larvae contain melanotic masses. (C-F) Double mutant combinations of homozygous Atg6 $6^{1}$ with ref(2)p (C), dif (D), dorsal (E) and Relish (F) do not suppress melanotic masses. (G) Triple mutant combination of Dif, rel and Atg6 ${ }^{1}$ showing presence of melanotic masses. $(\mathbf{H})$ dif, dorsal ${ }^{-/}$, Rel and Atg $6^{1}$ mutants exhibit melanotic masses. $n \geq 10$ for each genotype. Scale bars: $250 \mu \mathrm{m}$

melanotic blood cell masses in Drosophila (Minakhina and Steward, 2006). Therefore, we investigated whether mutations in either ref(2)P (Drosophila p62) or different combinations of NFkB genes suppress melanotic blood cell mass formation in homozygous $\operatorname{Atg} 6^{1}$ mutant larvae. All homozygous $\operatorname{Atg} 6^{1}$ mutant larvae possessed melanotic blood cell masses (black masses), whereas control larvae did not contain such structures (Fig. 6A,B). Significantly, homozygous ref(2)P mutants failed to suppress the Atg6 mutant melanotic mass phenotype (Fig. 6C). Drosophila has three NFKB proteins named Dif, Dorsal and Relish. Double mutant analyses of homozygous $\operatorname{Atg} 6^{1}$ with either homozygous dif, dorsal or Relish mutants indicated that mutations in each individual NFkB failed to suppress the Atg6 mutant melanotic mass phenotype (Fig. 6D-F). Therefore, we constructed a triple mutant containing homozygous dif, Relish and Atg6, and mutation of these two NFкB genes failed to suppress the formation of melanotic masses associated with $\operatorname{Atg} 6^{1}$ (Fig. 6G). Finally, we constructed a strain containing homozygous dif, Relish and Atg6 and also lacking one allele of dorsal (loss of all four genes was lethal at an earlier developmental stage), and this combination of $\mathrm{NF \kappa B}$ mutations failed to suppress the $\operatorname{Atg} 6^{1}$ melanotic blood cell mass phenotype (Fig. $6 \mathrm{H}$ ). These results suggest that neither $\operatorname{Ref}(2) \mathrm{P}$ nor NFKB proteins play a role in melanotic blood cell mass formation in Atg6 mutants.

To gain insight into the kinetics of melanotic blood cell mass formation, we followed the lymph gland (the larval hematopoietic organ) and melanotic mass development. We used hml $\Delta-G A L 4$ to drive GFP in blood cells in control $(n=107)$ and homozygous $\operatorname{Atg} 6^{1}$ mutant $(n=157)$ larvae. Compared with synchronized control animals, homozygous $\operatorname{Atg} 6^{1}$ mutant larvae displayed enlarged lymph glands (yellow boxes) between 80 hours and 104 hours after egg lay (Fig. 7A,B,E). During the same developmental interval, homozygous $\operatorname{Atg} 6^{l}$ mutant larvae progressively accumulated GFPpositive blood cell masses, whereas control animals lacked these structures. By 126 hours, all of the $\operatorname{Atg} 6^{l}$ mutant larvae contained numerous ectopic blood cell masses that were absent in controls 

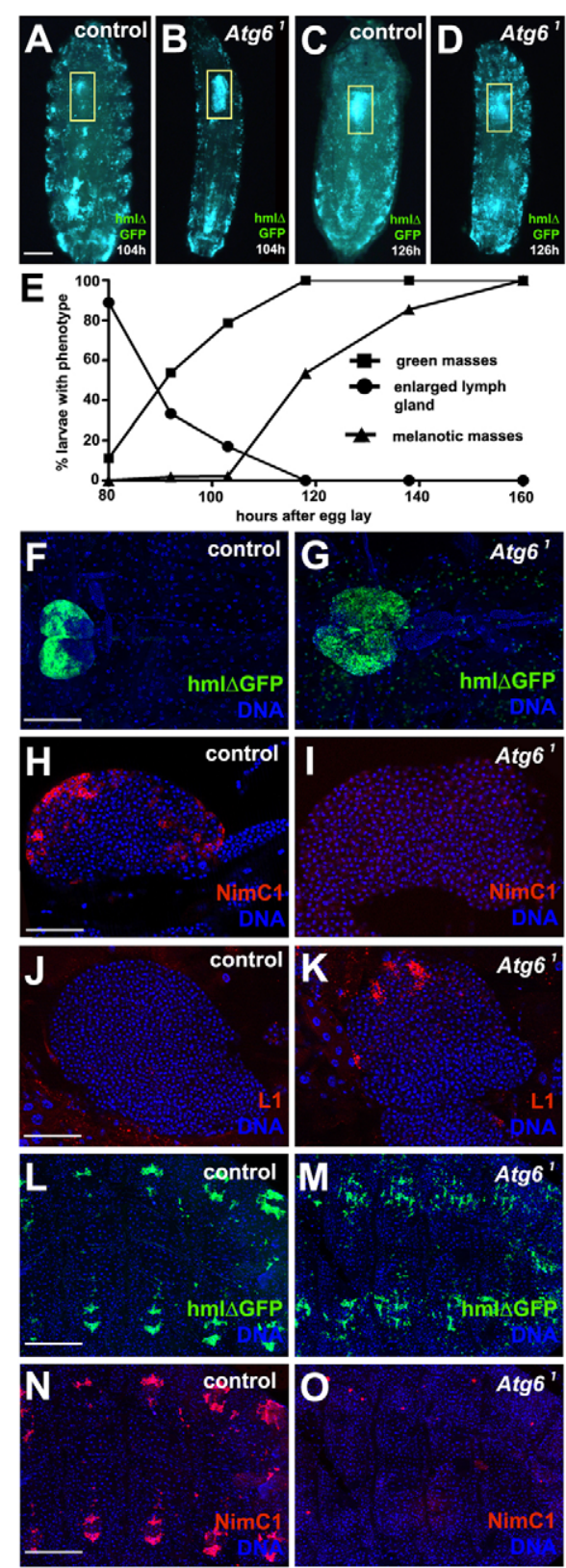

(Fig. 7C,D,E). These $\operatorname{Atg} 6^{1}$ mutant animal blood cell masses initiated melanization 104 hours after egg lay (following the formation of GFP-positive blood cell aggregates) and continued to do so until 160 hours after egg lay (Fig. 7E). Whereas control animals formed prepupae by 120 hours after egg lay, most $\operatorname{Atg} 6^{1}$ mutants did not pupariate.

We analyzed the lymph glands of age-matched control and homozygous $\operatorname{Atg} 6^{1}$ mutant third instar larval animals to obtain a better understanding of how hematopoiesis is altered. Homozygous $\operatorname{Atg} 6^{1}$ mutant lymph glands were larger than those of control animals (Fig. 7F,G; supplementary material Fig. S5). As expected, the plasmatocyte-specific blood cell differentiation marker NimrodC1 (NimC1) could be detected in the cortical zone in control lymph gland of control animals (Fig. 7H), but this marker could not be detected in the lymph glands of homozygous $\operatorname{Atg} 6^{1}$ mutant animals (Fig. 7I). L1 is a lamellocyte-specific blood cell differentiation marker that we did not detect in the cortical zone of control animal lymph glands (Fig. 7J). By contrast, homozygous $\operatorname{Atg} 6^{1}$ mutant lymph glands
Fig. 7. Loss of Atg6 leads to enlargement of lymph gland and altered blood cell development. (A) Control larva at 104 hours after egg lay expressing GFP in blood cells. (B) Homozygous Atg $6^{1}$ mutant larva at 104 hours after egg lay expressing GFP in blood cells. Note the enlarged lymph gland (yellow box). (C) Same control animal as in A at 126 hours after egg lay has formed a prepupa. (D) Same Atg6 ${ }^{1}$ mutant animal as in B at 126 hours after egg lay did not pupariate, and appeared to have an increased number of circulating blood cells. (E) Graph showing progression of different phenotypes exhibited by Atg 6 mutants during larval development. The larval stage-specific numbers of teeth on mouth hooks of control and Atg6 mutant larvae were used to normalize development of these genotypes. As larvae progress through development there is an increase in formation of blood cell aggregates followed by melanotic masses $(n=157)$. (F) Control $h m / \Delta-G A L 4$ UAS-GFP lymph gland at third instar larval stage $(n=10)$. (G) Atg6 mutant $h m / \Delta$ GAL4 UAS-GFP lymph gland at third instar stage $(n=10)$. (H) Control third instar lymph gland stained for NimrodC1 (NimC1) showing expression in the cortical zone ( $n=7)$. (I) Atg6 1 mutant third instar lymph gland stained for NimC1 showing a complete lack of expression in the cortical zone ( $n=15)$. (J) Control third instar larval lymph gland stained for lamellocyte specific antigen L1 showing no expression in the cortical zone $(n=7)$. (K) Atg6 $6^{1}$ mutant third instar animal lymph gland with increased expression of L1 in the cortical zone ( $n=7)$. (L) Control $h m / \triangle-G A L 4$ UASGFP animal showing sessile blood cells that are located in a reiterated pattern along abdominal segments $(n=7)$. (M) $h m / \Delta$-GAL4 UAS-GFPexpressing Atg $6^{1}$ mutant animals possess less patterned sessile blood cells along the abdominal segments than controls $(n=7)$. (N) NimC1 staining of animal shown in L. (0) NimC1 staining of animal shown in M indicates that this blood cell antigen is missing in sessile blood cells. Yellow boxes in A-D delineate lymph glands. Scale bars: $250 \mu \mathrm{m}$ in A; 100 $\mu \mathrm{m}$ in $\mathrm{F} ; 50 \mu \mathrm{m}$ in $\mathrm{H}, \mathrm{J} ; 200 \mu \mathrm{m}$ in L,N.

displayed increased L1 staining (Fig. 7K). In addition, blood cells that are present in clusters in abdominal segments expressed $\mathrm{NimC} 1$ in control animals, whereas those in $\operatorname{Atg} 6^{1}$ mutant animals appeared to lack the NimC1 antigen (Fig. 7L-O). Taken together, these data indicate that Atg6 plays an important role in blood cell development, and that an altered differentiation program probably contributes to melanotic blood cell masses.

\section{DISCUSSION}

Here we describe the genetic characterization of Atg6 in Drosophila. Atg6 is an essential gene, and most homozygous Atg6 null mutant animals die at the end of larval development. Drosophila lacking Atg6 function possess melanotic blood cell masses, as well as defects in several vesicle trafficking pathways.

Atg6 is a core component of the Vps34 complex. Studies in yeast and mammalian systems have identified Vps34 as an essential protein regulating a wide variety of vesicular trafficking events, including autophagy, endocytosis, and anterograde and retrograde transport between Golgi and the lysosome (Lindmo and Stenmark, 2006). Therefore, it is logical that Atg6 mutant cells not only have a defect in starvation-induced autophagy, but also fail to produce PI3P and have defects in endocytosis and protein secretion. Our data are consistent with reports from other animal systems in which beclin 1 mutants exhibit endocytosis defects (Ruck et al., 2011; Thoresen et al., 2010).

The accumulation of an endocytic tracer at the periphery of homozygous Atg6 mutant cells suggests that Atg6 functions at an early step of endocytosis. This conclusion is supported by the similarity between Atg6 and ESCRT II and III endocytic tracer phenotypes in the fat body. Therefore, it is possible that loss of Atg6 is similar to ESCRT mutants in flies, and that the Vps34 complex 
regulates receptor downregulation because of similar defects in endocytosis (Herz et al., 2006; Herz et al., 2009; Thompson et al., 2005; Vaccari and Bilder, 2005; Vaccari et al., 2009). However, the lack of an obvious Atg6 mutant eye over-growth phenotype suggests that Atg6 mutants are different from ESCRT pathway mutants. In addition, unlike ESCRT mutants, Atg6 mutant cell clones neither accumulated Notch intracellular domain antigen nor possessed alter the expression of the $m 2.61$ lac $Z$ Notch reporter (data not shown). An alternative explanation for the difference between Atg6 and ESCRT mutants is that maternally contributed Atg6 mRNA may enable normal Atg6 mutant eye imaginal disc development. Although loss of Atg6 suppresses ESCRT mutant developing eye tissue size, the pattern of these structures remains disrupted, suggesting that some aspects of the ESCRT phenotype cannot be suppressed. It is tempting to speculate that Atg6 functions at an earlier stage than these ESCRT genes in endocytosis, but additional studies are needed to understand the relationship of these factors during endocytosis.

Recent work indicates that autophagy regulates protein secretion (Deretic et al., 2012). To our knowledge, this is the first report of Atg6 regulating protein secretion. In addition, we show that loss of either Vps34 or Atg1 in salivary gland cells also leads to disruption of protein secretion. Therefore, our data indicate that protein secretion might be an autophagy-dependent process. Interestingly, we noted differences in the size of Sgs3-GFP vesicles in Atg6, Vps34 and Atg1 mutant cells (Fig. 4E-G'). Although these genes might function at distinct steps in the maturation of secretory vesicles, it is also possible that differences in maternal contribution of mRNAs in these mutants are responsible for the differences in these mutant phenotypes. Beclin 1 and PI3P localize to the trans Golgi network (Gillooly et al., 2000; Kihara et al., 2001a). Thus, it is also possible that Vps34 and Atg6 are part of a third Vps34 complex that can regulate protein secretion, although it is also possible that the Vps34 complex that regulates autophagy participates in this process.

Atg6 mutant larvae possess excess hemocytes, the Drosophila equivalent of macrophages, and contain melanotic blood cell masses prior to their death. Melanotic masses are thought to be caused by at least two possible mechanisms: (1) tissue damage that recruits blood cells to encapsulate the unhealthy tissue and potentially protect the organism, and (2) over-proliferation of the blood cell lineage due to a defect in the hematopoietic stem cell niche (Minakhina and Steward, 2006). In support of the latter possibility, a recent study showed that hemocytes with decreased autophagy have decreased recruitment to epidermal wounds because of impaired cortical remodeling in the blood cells (Kadandale et al., 2010). Although blood cells clearly surround the melanotic masses in Atg6 mutant larvae, it is unclear whether the masses themselves are composed strictly of hemocytes and whether the masses result from hemocyte over-proliferation, or if hemocytes are induced to proliferate by the presence of melanotic masses. Our data indicate that Atg6 mutants have enlarged hematopoietic organs, more blood cells and altered blood cell differentiation and that blood cell aggregations precede the formation of melanotic masses. However, we cannot exclude the possibility that cells that are not of hematopoietic origin are involved in the initiation of melanotic masses.

It is interesting that, like beclin 1 mutant mice, loss of Atg6 in Drosophila results in expansion of the hematopoietic lineage. NF$\kappa \mathrm{Bs}$ are known to regulate hematopoiesis in both Drosophila and mammals. In flies, Toll and cactus are key regulators of NF- $\kappa \mathrm{B}$ signaling, and either Toll gain-of-function or cactus loss-of-function mutants lead to over-proliferation of hemocytes, in particular lamellocytes, resulting in the formation of melanotic masses (Qiu et al., 1998). Given the connection between beclin 1, p62 and NF- $\kappa B$ (Mathew et al., 2009), we speculated that the melanotic mass phenotype in Atg6 mutants could be due to misregulation of p62

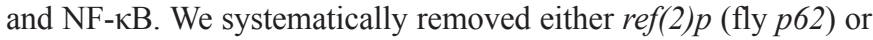
the three Drosophila NF- $\mathrm{BBs}$ dorsal, dif and Rel in combination with loss of Atg6. Surprisingly, mutations in these genes failed to suppress the Atg6 melanotic mass phenotype.

Numerous reports indicate that beclin 1 plays an important role in cancer, and most studies attribute this function to a defect in autophagy (White and DiPaola, 2009). Although autophagy is likely to contribute to tumor progression, it is also possible that the influence of beclin 1 on other vesicle trafficking pathways may promote tumor development. Consistent with this possibility, loss of Atg5 and autophagy leads to benign adenomas in livers that fail to cause cancer, but this phenotype is not observed in other tissues (Takamura et al., 2011). Here, we show that Atg6 influences multiple trafficking pathways in flies, and that Atg6 mutant animals possess hematopoietic defects and melanotic blood cell masses. Future studies of Drosophila Atg6 mutants should resolve a number of questions that are relevant to both the fundamental cellular function of this protein, as well as potentially advance our understanding of how beclin 1 functions as a tumor suppressor.

\section{Acknowledgements}

We thank Y. Rong for advice about gene targeting; C. Evans and U. Banerjee for advice about lymph gland analyses; I. Ando, A. Bergmann, D. Bilder, M. Freeman, T. Ip, T. Neufeld, I. Nezis, N. Silverman, H. Stenmark, the Bloomington Stock Center and the Vienna Drosophila RNAi Center for flies and antibodies; T. Fortier for technical support; and the Baehrecke lab for constructive comments.

\section{Funding}

This work was supported by National Institutes of Health grants [CA159314 to E.H.B. and S10RR027897 to the UMass EM Core]. E.H.B. is an Ellison Medical Foundation Scholar and a member of the UMass DERC [DK32520]. Deposited in PMC for release after 12 months.

\section{Author contributions}

B.V.S., J.H.H. and E.H.B. designed experiments; B.V.S., J.H.H. and C.M.P. performed experiments; and all authors wrote and discussed the manuscript.

\section{Competing interests statement}

The authors declare no competing financial interests.

\section{Supplementary material}

Supplementary material available online at http://dev.biologists.org/lookup/suppl/doi:10.1242/dev.089490/-/DC1

\section{References}

Aita, V. M., Liang, X. H., Murty, V. V., Pincus, D. L., Yu, W., Cayanis, E., Kalachikov, S., Gilliam, T. C. and Levine, B. (1999). Cloning and genomic organization of beclin 1, a candidate tumor suppressor gene on chromosome 17q21. Genomics 59, 59-65.

Asha, H., Nagy, I., Kovacs, G., Stetson, D., Ando, I. and Dearolf, C. R. (2003). Analysis of Ras-induced overproliferation in Drosophila hemocytes. Genetics $163,203-215$

Berry, D. L. and Baehrecke, E. H. (2007). Growth arrest and autophagy are required for salivary gland cell degradation in Drosophila. Cell 131, 1137-1148.

Biyasheva, A., Do, T. V., Lu, Y., Vaskova, M. and Andres, A. J. (2001). Glue secretion in the Drosophila salivary gland: a model for steroid-regulated exocytosis. Dev. Biol. 231, 234-251.

Bodenstein, D. (1965). The postembryonic development of Drosophila. In Biology of Drosophila (ed. M. Demerec), pp. 275-367. New York, NY: Hafner Publishing.

Budnik, V., Gorczyca, M. and Prokop, A. (2006). Selected methods for the anatomical study of Drosophila embryonic and larval neuromuscular junctions. Int. Rev. Neurobiol. 75, 323-365.

Denton, D., Shravage, B., Simin, R., Mills, K., Berry, D. L., Baehrecke, E. H. and Kumar, S. (2009). Autophagy, not apoptosis, is essential for midgut cell death in Drosophila. Curr. Biol. 19, 1741-1746.

Deretic, V. (2011). Autophagy in immunity and cell-autonomous defense against intracellular microbes. Immunol. Rev. 240, 92-104. 
Deretic, V., Jiang, S. and Dupont, N. (2012). Autophagy intersections with conventional and unconventional secretion in tissue development remodeling and inflammation. Trends Cell Biol. 22, 397-406.

Ellson, C. D., Andrews, S., Stephens, L. R. and Hawkins, P. T. (2002). The PX domain: a new phosphoinositide-binding module. J. Cell Sci. 115, 1099-1105.

Fimia, G. M., Stoykova, A., Romagnoli, A., Giunta, L., Di Bartolomeo, S., Nardacci, R., Corazzari, M., Fuoco, C., Ucar, A., Schwartz, P. et al. (2007) Ambra1 regulates autophagy and development of the nervous system. Nature 447, 1121-1125.

Funderburk, S. F., Wang, Q. J. and Yue, Z. (2010). The Beclin 1-VPS34 complex at the crossroads of autophagy and beyond. Trends Cell Biol. 20, 355-362.

Gillooly, D. J., Morrow, I. C., Lindsay, M., Gould, R., Bryant, N. J., Gaullier, J. M., Parton, R. G. and Stenmark, H. (2000). Localization of

phosphatidylinositol 3-phosphate in yeast and mammalian cells. EMBO J. 19, 4577-4588.

Harding, T. M., Morano, K. A., Scott, S. V. and Klionsky, D. J. (1995). Isolation and characterization of yeast mutants in the cytoplasm to vacuole protein targeting pathway. J. Cell Biol. 131, 591-602

Henne, W. M., Buchkovich, N. J. and Emr, S. D. (2011). The ESCRT pathway. Dev. Cell 21, 77-91.

Herz, H. M., Chen, Z., Scherr, H., Lackey, M., Bolduc, C. and Bergmann, A. (2006). vps25 mosaics display non-autonomous cell survival and overgrowth and autonomous apoptosis. Development 133, 1871-1880.

Herz, H. M., Woodfield, S. E., Chen, Z., Bolduc, C. and Bergmann, A. (2009). Common and distinct genetic properties of ESCRT-II components in Drosophila. PLOS ONE 4, e4165.

Itakura, E., Kishi, C., Inoue, K. and Mizushima, N. (2008). Beclin 1 forms two distinct phosphatidylinositol 3-kinase complexes with mammalian Atg14 and UVRAG. Mol. Biol. Cell 19, 5360-5372.

Juhász, G., Hill, J. H., Yan, Y., Sass, M., Baehrecke, E. H., Backer, J. M. and Neufeld, T. P. (2008). The class III PI(3)K Vps34 promotes autophagy and endocytosis but not TOR signaling in Drosophila. J. Cell Biol. 181, 655-666.

Kadandale, P., Stender, J. D., Glass, C. K. and Kiger, A. A. (2010). Conserved role for autophagy in Rho1-mediated cortical remodeling and blood cell recruitment. Proc. Natl. Acad. Sci. USA 107, 10502-10507.

Karantza-Wadsworth, V., Patel, S., Kravchuk, O., Chen, G., Mathew, R., Jin, S. and White, E. (2007). Autophagy mitigates metabolic stress and genome damage in mammary tumorigenesis. Genes Dev. 21, 1621-1635.

Kihara, A., Kabeya, Y., Ohsumi, Y. and Yoshimori, T. (2001a). Beclinphosphatidylinositol 3-kinase complex functions at the trans-Golgi network. EMBO Rep. 2, 330-335

Kihara, A., Noda, T., Ishihara, N. and Ohsumi, Y. (2001 b). Two distinct Vps34 phosphatidylinositol 3-kinase complexes function in autophagy and carboxypeptidase Y sorting in Saccharomyces cerevisiae. J. Cell Biol. 152, 519530

Kirisako, T., Baba, M., Ishihara, N., Miyazawa, K., Ohsumi, M., Yoshimori, T., Noda, T. and Ohsumi, Y. (1999). Formation process of autophagosome is traced with Apg8/Aut7p in yeast. J. Cell Biol. 147, 435-446.

Lebestky, T., Chang, T., Hartenstein, V. and Banerjee, U. (2000). Specification of Drosophila hematopoietic lineage by conserved transcription factors. Science 288, 146-149.

Liang, C., Lee, J. S., Inn, K. S., Gack, M. U., Li, Q., Roberts, E. A., Vergne, I., Deretic, V., Feng, P., Akazawa, C. et al. (2008). Beclin1-binding UVRAG targets the class $C$ Vps complex to coordinate autophagosome maturation and endocytic trafficking. Nat. Cell Biol. 10, 776-787.

Lindmo, K. and Stenmark, H. (2006). Regulation of membrane traffic by phosphoinositide 3-kinases. J. Cell Sci. 119, 605-614.

Lindmo, K., Brech, A., Finley, K. D., Gaumer, S., Contamine, D., Rusten, T. E. and Stenmark, H. (2008). The PI 3-kinase regulator Vps15 is required for autophagic clearance of protein aggregates. Autophagy 4, 500-506.

Maroni, G. and Stamey, S. C. (1983). Use of blue food to select synchronous, late third instar larvae. Dros. Inf. Serv. 59, 142-143.

Mathew, R., Kongara, S., Beaudoin, B., Karp, C. M., Bray, K., Degenhardt, K., Chen, G., Jin, S. and White, E. (2007). Autophagy suppresses tumor progression by limiting chromosomal instability. Genes Dev. 21, 1367-1381.

Mathew, R., Karp, C. M., Beaudoin, B., Vuong, N., Chen, G., Chen, H. Y., Bray, K., Reddy, A., Bhanot, G., Gelinas, C. et al. (2009). Autophagy suppresses tumorigenesis through elimination of p62. Cell 137, 1062-1075.

Matsunaga, K., Saitoh, T., Tabata, K., Omori, H., Satoh, T., Kurotori, N., Maejima, I., Shirahama-Noda, K., Ichimura, T., Isobe, T. et al. (2009). Two Beclin 1-binding proteins, Atg14L and Rubicon, reciprocally regulate autophagy at different stages. Nat. Cell Biol. 11, 385-396.

Minakhina, S. and Steward, R. (2006). Melanotic mutants in Drosophila: pathways and phenotypes. Genetics 174, 253-263.

Mizushima, N. and Komatsu, M. (2011). Autophagy: renovation of cells and tissues. Cell 147, 728-741.

Muro, I., Berry, D. L., Huh, J. R., Chen, C. H., Huang, H., Yoo, S. J., Guo, M., Baehrecke, E. H. and Hay, B. A. (2006). The Drosophila caspase Ice is important for many apoptotic cell deaths and for spermatid individualization, a nonapoptotic process. Development 133, 3305-3315.
Nezis, I. P., Simonsen, A., Sagona, A. P., Finley, K., Gaumer, S., Contamine, D., Rusten, T. E., Stenmark, H. and Brech, A. (2008). Ref(2)P, the Drosophila melanogaster homologue of mammalian p62, is required for the formation of protein aggregates in adult brain. J. Cell Biol. 180, 1065-1071.

Qiu, P., Pan, P. C. and Govind, S. (1998). A role for the Drosophila Toll/Cactus pathway in larval hematopoiesis. Development 125, 1909-1920.

Qu, X., Yu, J., Bhagat, G., Furuya, N., Hibshoosh, H., Troxel, A., Rosen, J., Eskelinen, E. L., Mizushima, N., Ohsumi, Y. et al. (2003). Promotion of tumorigenesis by heterozygous disruption of the beclin 1 autophagy gene. J. Clin. Invest. 112, 1809-1820.

Rong, Y. S. and Golic, K. G. (2000). Gene targeting by homologous recombination in Drosophila. Science 288, 2013-2018.

Ruck, A., Attonito, J., Garces, K. T., Núnez, L., Palmisano, N. J., Rubel, Z., Bai, Z., Nguyen, K. C., Sun, L., Grant, B. D. et al. (2011). The Atg6/Vps30/Beclin 1 ortholog BEC-1 mediates endocytic retrograde transport in addition to autophagy in C. elegans. Autophagy 7, 386-400.

Scott, R. C., Schuldiner, O. and Neufeld, T. P. (2004). Role and regulation of starvation-induced autophagy in the Drosophila fat body. Dev. Cell 7, 167-178.

Scott, R. C., Juhász, G. and Neufeld, T. P. (2007). Direct induction of autophagy by Atg1 inhibits cell growth and induces apoptotic cell death. Curr. Biol. 17, 111.

Seaman, M. N., Marcusson, E. G., Cereghino, J. L. and Emr, S. D. (1997). Endosome to Golgi retrieval of the vacuolar protein sorting receptor, Vps 10p requires the function of the VPS29, VPS30, and VPS35 gene products. J. Cell Biol. 137, 79-92.

Simonsen, A., Birkeland, H. C., Gillooly, D. J., Mizushima, N., Kuma, A., Yoshimori, T., Slagsvold, T., Brech, A. and Stenmark, H. (2004). Alfy, a novel FYVE-domain-containing protein associated with protein granules and autophagic membranes. J. Cell Sci. 117, 4239-4251.

Stenmark, H., Aasland, R. and Driscoll, P. C. (2002). The phosphatidylinositol $3-$ phosphate-binding FYVE finger. FEBS Lett. 513, 77-84

Takahashi, Y., Coppola, D., Matsushita, N., Cualing, H. D., Sun, M., Sato, Y., Liang, C., Jung, J. U., Cheng, J. Q., Mulé, J. J. et al. (2007). Bif-1 interacts with Beclin 1 through UVRAG and regulates autophagy and tumorigenesis. Nat. Cell Biol. 9, 1142-1151.

Takamura, A., Komatsu, M., Hara, T., Sakamoto, A., Kishi, C., Waguri, S., Eishi, Y., Hino, O., Tanaka, K. and Mizushima, N. (2011). Autophagydeficient mice develop multiple liver tumors. Genes Dev. 25, 795-800.

Thompson, B. J., Mathieu, J., Sung, H. H., Loeser, E., Rørth, P. and Cohen, S. M. (2005). Tumor suppressor properties of the ESCRT-II complex component Vps25 in Drosophila. Dev. Cell 9, 711-720.

Thoresen, S. B., Pedersen, N. M., Liestøl, K. and Stenmark, H. (2010). A phosphatidylinositol 3-kinase class III sub-complex containing VPS15, VPS34, Beclin 1, UVRAG and BIF-1 regulates cytokinesis and degradative endocytic traffic. Exp. Cell Res. 316, 3368-3378.

Thumm, M., Egner, R., Koch, B., Schlumpberger, M., Straub, M., Veenhuis, M. and Wolf, D. H. (1994). Isolation of autophagocytosis mutants of Saccharomyces cerevisiae. FEBS Lett. 349, 275-280.

Tsukada, M. and Ohsumi, Y. (1993). Isolation and characterization of autophagy-defective mutants of Saccharomyces cerevisiae. FEBS Lett. 333, 169-174.

Vaccari, T. and Bilder, D. (2005). The Drosophila tumor suppressor vps 25 prevents nonautonomous overproliferation by regulating notch trafficking Dev. Cell 9, 687-698.

Vaccari, T., Rusten, T. E., Menut, L., Nezis, I. P., Brech, A., Stenmark, H. and Bilder, D. (2009). Comparative analysis of ESCRT-I, ESCRT-II and ESCRT-III function in Drosophila by efficient isolation of ESCRT mutants. J. Cell Sci. 122, 2413-2423.

White, E. and DiPaola, R. S. (2009). The double-edged sword of autophagy modulation in cancer. Clin. Cancer Res. 15, 5308-5316.

Wu, J., Randle, K. E. and Wu, L. P. (2007). ird1 is a Vps15 homologue important for antibacterial immune responses in Drosophila. Cell. Microbiol. 9, 1073-1085.

Wucherpfennig, T., Wilsch-Bräuninger, M. and González-Gaitán, M. (2003). Role of Drosophila Rab5 during endosomal trafficking at the synapse and evoked neurotransmitter release. J. Cell Biol. 161, 609-624.

Xu, T. and Rubin, G. M. (1993). Analysis of genetic mosaics in developing and adult Drosophila tissues. Development 117, 1223-1237.

Yang, Z., Huang, J., Geng, J., Nair, U. and Klionsky, D. J. (2006). Atg22 recycles amino acids to link the degradative and recycling functions of autophagy. Mol. Biol. Cell 17, 5094-5104.

Yue, Z., Jin, S., Yang, C., Levine, A. J. and Heintz, N. (2003). Beclin 1, an autophagy gene essential for early embryonic development, is a haploinsufficient tumor suppressor. Proc. Natl. Acad. Sci. USA 100, 15077-15082.

Zhang, Y., Qi, H., Taylor, R., Xu, W., Liu, L. F. and Jin, S. (2007). The role of autophagy in mitochondria maintenance: characterization of mitochondrial functions in autophagy-deficient S. cerevisiae strains. Autophagy 3, 337-346.

Zhong, Y., Wang, Q. J., Li, X., Yan, Y., Backer, J. M., Chait, B. T., Heintz, N. and Yue, Z. (2009). Distinct regulation of autophagic activity by Atg14L and Rubicon associated with Beclin 1-phosphatidylinositol-3-kinase complex. Nat. Cell Biol. 11, 468-476. 